\title{
New estimates of the contribution of Wolf-Rayet stellar winds to the Galactic ${ }^{26} \mathrm{Al}$
}

\author{
A. Palacios ${ }^{1}$, G. Meynet ${ }^{2}$, C. Vuissoz ${ }^{3}$, J. Knödlseder ${ }^{4}$, D. Schaerer ${ }^{2,5}$, M. Cerviño $^{6}$, and N. Mowlavi ${ }^{7}$ \\ ${ }^{1}$ Institut d'Astronomie et d'Astrophysique de l'Université Libre de Bruxelles, 226 Bd du Triomphe, \\ 1050 Brussels, Belgium \\ e-mail: palacios@astro.ulb.ac.be \\ 2 Observatoire de Genève, 51 Chemin des Maillettes, 1290 Sauverny, Switzerland \\ e-mail: Georges . Meynet@obs .unige.ch \\ 3 Laboratoire d'Astrophysique de l'EPFL - Observatoire, 1290 Chavannes-des-Bois, Switzerland \\ ${ }^{4}$ Centre d'Étude Spatiale des Rayonnements - 9, Av. du Colonel Roche, BP 4346, 31028 Toulouse Cedex 4, France \\ 5 Laboratoire d'Astrophysique de Toulouse-Tarbes, 14 avenue Edouard Belin, 31400 Toulouse, France \\ ${ }^{6}$ Instituto de Astrofísica de Andalucía (CSIC) - Camino bajo de Huétor 24, Apdo. 3004, 18080 Granada, Spain \\ 7 INTEGRAL Science Data Center, Chemin d'Ecogia 16, 1290 Versoix, Switzerland
}

Received 30 July 2004 / Accepted 13 August 2004

\begin{abstract}
We present new yields of ${ }^{26} \mathrm{Al}$ from Wolf-Rayet stellar winds based on rotating stellar models which account well for numerous observed properties of massive stars. We study the impacts on the yields of a change of initial mass, metallicity and initial rotation velocity. We also consider the effects of a change of mass loss rates during the Wolf-Rayet phase.

We show that for surface rotation velocities during the core H-burning phase matching to the observed ones, the quantity of ${ }^{26} \mathrm{Al}$ ejected by a star of a given initial mass and metallicity is roughly doubled when the effects of rotation are taken into account. The metallicity dependence of the yield is, on the other hand, very similar to that obtained from non-rotating models. We estimate that at least about $20 \%$ to $50 \%$ (e.g. $\sim 0.6-1.4 M_{\odot}$ ) of the live ${ }^{26} \mathrm{Al}$ detected in the Milky-Way originates from Wolf-Rayet stellar winds. We show the importance of a good knowledge of the present metallicity gradient and star formation rate in our galaxy for modeling both the variation of the ${ }^{26} \mathrm{Al}$ surface density with the galactocentric distance and the global contribution of the Wolf-Rayet stellar winds to the present galactic mass of ${ }^{26} \mathrm{Al}$.
\end{abstract}

Key words. nuclear reactions, nucleosynthesis, abundances - stars: rotation - Galaxy: abundances - stars: Wolf-Rayet stars: abundances

\section{Introduction}

The ${ }^{26} \mathrm{Al}$ radionuclide, live or extinct, is observed in at least three different types of media: 1) excesses of daughter elements from ${ }^{26} \mathrm{Al}$ are observed in presolar stardust grains (see the review by Clayton \& Nittler 2004); 2) evidence for injection of live ${ }^{26} \mathrm{Al}$ in the early Solar System nebula can be found in meteorites (see e.g. MacPherson et al. 1995); 3) ${ }^{26} \mathrm{Al}$ is also detected in the galactic interstellar medium through its decay emission line at 1.809 MeV (Mahoney et al. 1982; Diehl et al. 1995). This diffuse emission is observed in the plane of our Galaxy. From its total intensity galactic ${ }^{26} \mathrm{Al}$ masses have been derived in the range from $1.5 M_{\odot}$ to $3 M_{\odot}$, depending much on the assumed underlying ${ }^{26} \mathrm{Al}$ density distribution, and in particular, on the yet poorly determined scale height of ${ }^{26} \mathrm{Al}$ (see e.g. Fig. 4.7 in Knödlseder 1997). In this paper we shall focus our attention on the galactic interstellar ${ }^{26} \mathrm{Al}$.

Since this nuclide has a lifetime of roughly $10^{6} \mathrm{yr}$, much shorter than the Galaxy lifetime, it must be continuously produced by one or more nucleosynthetic source to be observed. The question which still remains to be solved is what is the nature of these sources. A correct answer to this question is not only interesting on its own, but will enable to use the emissivity at $1.809 \mathrm{MeV}$ as a penetrating tool ${ }^{1}$ for studying regions of current nucleosynthetic activity in the Milky Way.

From the observed distribution of the emission in the plane of our Galaxy it is generally inferred that the massive stars, either through their winds (Dearborn \& Blake 1985; Vuissoz et al. 2004) or through their ejections at the time of the supernova event (Timmes et al. 1995; Woosley \& Weaver 1995), are the main sources of ${ }^{26} \mathrm{Al}$ (see also the review by Prantzos \& Diehl 1996, and references therein). Here we shall only address the contribution of the winds of Wolf-Rayet (WR) stars.

\section{Wolf-Rayet versus core collapse supernovae}

Although WR stars end their lives as supernovae, and also contribute to the interstellar medium enrichment of ${ }^{26} \mathrm{Al}$ through explosive nucleosynthesis, we distinguish the

\footnotetext{
${ }^{1} \gamma$-rays do not suffer any absorption by the interstellar medium.
} 
"wind" contribution from that of the "supernova explosion" considering that observations allow us to disentangle the two channels for ${ }^{26} \mathrm{Al}$ production. Indeed the ejection of ${ }^{26} \mathrm{Al}$ by the winds of massive stars is not accompanied by the ejection of ${ }^{60} \mathrm{Fe}$, a radionuclide with a lifetime of $2.2 \mathrm{Myr}$, whereas its ejection at the time of the supernova explosion is. Secondly, after a burst of star formation, the enrichment of the interstellar medium by the WR stellar winds begins prior to the enrichment by the supernovae explosions (Cerviño et al. 2000; Plüschke et al. 2002). This is due to the fact that WR stars originate from more massive (and thus shorter lived) progenitors than the bulk of the core collapse supernovae (hereafter cc-SNae). Therefore observations of sufficiently young associations of massive stars, as the Cygnus region (Knödlseder et al. 2002), allow us in principle to disentangle WR wind and cc-SNae contributions.

Recently, Smith (2004a) has reported a first detection of the 1.117 and $1.332 \mathrm{MeV}$ gamma-ray lines attributed to the radioactive decay of ${ }^{60} \mathrm{Fe}$ from the inner Galaxy region, using the Reuven Ramaty High Energy Solar Spectroscopic Imager (RHESSI). After a more deeper analysis, he revised his first flux estimates downwards, to a marginal 2.6 sigma detection level (Smith 2004b). The observed flux in each of the ${ }^{60} \mathrm{Fe}$ line amounts to about $10 \%$ of the ${ }^{26} \mathrm{Al}$ flux, slightly smaller than the flux ratio predicted by Timmes et al. (1995) from calculations of type II supernovae ${ }^{2}$ (hereafter SNII) nucleosynthesis by Woosley \& Weaver (1995). Taken at face, such an agreement would mean that all the galactic ${ }^{26} \mathrm{Al}$ could originate from the SNII. However, as discussed in Prantzos (2004), more recent models of the evolution and nucleosynthesis of cc-SNae such as those of Rauscher et al. (2002) or those of Limongi \& Chieffi (2003), predict much higher ${ }^{60} \mathrm{Fe} /{ }^{26} \mathrm{Al}$ line flux ratios. These models seem thus to leave more room for sources, such as the WR stellar winds, ejecting ${ }^{26} \mathrm{Al}$ and not ${ }^{60} \mathrm{Fe}$. According to Prantzos (2004), with these new stellar yields, the RHESSI results can be recovered provided that at least half of ${ }^{26} \mathrm{Al}$ originates from WR stellar winds. There are at least three other arguments supporting some additional contributions to that of the supernovae. Firstly, the new supernovae yields appear too low to allow an injection rate of $2 M_{\odot}$ of ${ }^{26} \mathrm{Al}$ per $\mathrm{Myr}$ (Prantzos 2004). Secondly the observation of a bump of emissivity at $1.809 \mathrm{MeV}$ in the direction of the Cygnus region, a region with no sign of recent supernova activity, also points towards the existence of sources such as the WR stars, that eject ${ }^{26} \mathrm{Al}$ even before the first supernovae appear (Knödlseder et al. 2002). Finally, from the point of view of stellar evolution models, ${ }^{26} \mathrm{Al}$ enrichment of the interstellar medium by WR stars is an inherent and unavoidable consequence of the most massive stars evolution if their observed properties are to be reproduced (Meynet et al. 1997). An important contribution of WR stars to the galactic ${ }^{26} \mathrm{Al}$ is therefore unavoidable.

The purpose of the present work is to provide yields from WR stellar winds based on recent grids of rotating stellar models (Meynet \& Maeder 2003, 2005, hereafter Papers X and XI respectively). In these previous two papers we have

\footnotetext{
2 The mass of the progenitors of type II supernovae lies between $11 M_{\odot}$ and $40 M_{\odot}$, excluding WR stars in the case of non-rotating models.
}

investigated the effects of rotation on the formation of the WR stars at different metallicities. We showed that the inclusion of rotation makes the formation of WR stars easier, thus helping to reproduce the observed variations of the number ratio of WR to O-type stars as a function of the metallicity, of the ratio of $\mathrm{WC}$ to $\mathrm{WN}$ stars for metallicities below about $Z=0.020$ (solar metallicity), as well as the observed variation with the metallicity of the number fraction of type Ibc supernovae with respect to SNII (Prantzos \& Boissier 2003). Furthermore, models with rotation are able to account for the small (although significant) fraction of WR stars presenting at their surface both $\mathrm{H}$ - and He-burning products (see e.g. Crowther et al. 1995). None of these observed features can be fitted by non-rotating stellar models computed with recent mass loss rates prescriptions (see Sect. 2).

Despite the fact that the rotating models well reproduce the mentioned above observed features as well as the observed surface enrichments (Heger \& Langer 2000; Meynet \& Maeder 2000, hereafter Paper V) and the number ratio of blue to red supergiants in the Small Magellanic Cloud (Maeder \& Meynet 2001; hereafter Paper VII), the rotating models have some difficulties in accounting for the observed fraction of WC to WN stars at high metallicity. Typically, rotating models seem to underestimate this ratio at twice the solar metallicity by about a factor of two. In Paper XI we attributed this remaining discrepancy as due to one (or both) of the following two causes:

1) The incompleteness of the observed sample in high metallicity regions might bias the result. This seems already to be the case at solar metallicity where according to Massey \& Johnson (1998) the observed WC/WN ratio is overestimated. The less luminous WC stars are more difficult to detect than the more luminous WN stars, especially in regions where the extinction may be high. In contrast, in the Magellanic Clouds, where the models well reproduce the observed ratio, the stars have nearly all the same metallicity, are at the same distance and the internal extinction is low.

2) The mass loss rates during the post H-burning $\mathrm{WN}$ phase are underestimated. This would increase the WC phase duration at the expense of the $\mathrm{WN}$ phase. Let us note that even if the mass loss rates would be underestimated in the post H-burning WN phases, this would not deeply modify the present results as far as ${ }^{26} \mathrm{Al}$ is concerned.

Despite the above remaining difficulty, we think that the present models can sufficiently well account for the observed properties of massive stars to allow an acceptable estimate of their ${ }^{26} \mathrm{Al}$ yields. In Sect. 2 we briefly recall the physical ingredients of the models. The effects of rotation on ${ }^{26} \mathrm{Al}$ production by WR stars at various metallicities are discussed in Sect. 3. A small subset of the present models for the metallicities at $Z=0.020$ and 0.040 were already briefly discussed in Vuissoz et al. (2004). An estimate of the global contribution of WR stars to the ${ }^{26} \mathrm{Al}$ present in the Milky Way is given in Sect. 4, while Sect. 5 presents the main conclusions of this work. 


\section{Physical ingredients of the models}

The grid of models presented here was computed with the Geneva stellar evolution code. The physical ingredients are essentially the same as in Papers X and XI, except for the inclusion of the $\mathrm{Ne}-\mathrm{Na}$ and $\mathrm{Mg}-\mathrm{Al}$ reaction chains and the omission of the effects of the wind anisotropies induced by rotation (see below). Let us briefly recall the main points relevant for ${ }^{26} \mathrm{Al}$ production:

- The initial compositions are adapted for the different metallicities considered here. For a given metallicity $Z$ (in mass fraction), the initial helium mass fraction $Y$ is given by the relation $Y=Y_{\mathrm{p}}+\Delta Y / \Delta Z \cdot Z$, where $Y_{\mathrm{p}}$ is the primordial helium abundance and $\Delta Y / \Delta Z$ the slope of the helium-to-metal enrichment law. We use the same values as in Paper VII i.e. $Y_{\mathrm{p}}=0.23$ and $\Delta Y / \Delta Z=2.5$. For the metallicities $Z=0.004,0.008$ and 0.040 considered in this work, we thus have $X=0.757,0.744,0.640$ and $Y=0.239$, $0.248,0.320$ respectively. For the mixture of the heavy elements, we adopt the same mixture as the one used to compute the opacity tables for solar composition (Iglesias \& Rogers 1996).

- Since mass loss rates are a key ingredient of the models in the mass range considered here, let us recall the prescriptions used. The changes of the mass loss rates $\dot{M}$ with rotation are taken into account as explained in Maeder \& Meynet (2000a, hereafter Paper VI). As reference, we adopt the mass loss rates of Vink et al. $(2000,2001)$ who account for the occurrence of bi-stability limits which change the wind properties and mass loss rates. For the domain not covered by these authors we use the results by de Jager et al. (1988). For the non-rotating models, since the empirical values for the mass loss rates are based on stars covering the whole range of rotational velocities, we must apply a reduction factor to the empirical rates to make them correspond to the non rotating case. We used a value of 0.85 , as in Paper VII. During the WR phase we use the mass loss rates by Nugis \& Lamers (2000). These rates, which account for the clumping effects in the winds, are smaller by a factor 2-3 compared to the ones used in our previous non-rotating "enhanced mass loss rate" stellar grids with predictions of ${ }^{26} \mathrm{Al}$ yields (Meynet et al. 1997).

- During the non-WR phases of the present models, we assumed that the mass loss rates depend on the initial metallicity as $\dot{M}(Z)=\left(Z / Z_{\odot}\right)^{1 / 2} \dot{M}\left(Z_{\odot}\right)$ (Kudritzki \& Puls 2000; Vink et al. 2001), while during the WR phase we assumed no metallicity dependence. At $Z=0.040$, we also computed a series of models with metallicity dependent mass loss rates during the WR phase. According to Crowther et al. (2002), mass loss rates during the WR phase may show the same metallicity dependence as the winds of O-type stars, i.e. scale with $\sim\left(Z / Z_{\odot}\right)^{1 / 2}$.

- A moderate overshooting is included in the present rotating and non-rotating models. The radii of the convective cores are increased with respect to their values obtained by the Schwarzschild criterion by a quantity equal to $0.1 H_{\mathrm{p}}$, where $H_{\mathrm{p}}$ is the pressure scale height estimated at the Schwarzschild boundary.
- The effect of rotation on the transport of the chemical species and of the angular momentum are accounted for as in Papers VII and VIII (Meynet \& Maeder 2002). All the models were computed up to the end of the He-burning phase.

- As initial rotation, we have considered a value equal to $300 \mathrm{~km} \mathrm{~s}^{-1}$ on the ZAMS for all the initial masses and metallicities considered. At solar metallicity, this initial value produces time averaged equatorial velocities on the main sequence (hereafter MS) well in the observed range, i.e. between 200 and $250 \mathrm{~km} \mathrm{~s}^{-1}$. At low metallicities this initial rotational velocity corresponds also to mean values between 200 and $250 \mathrm{~km} \mathrm{~s}^{-1}$ on the MS, while at twice the solar metallicity, the mean velocity is lower, between 160 and $230 \mathrm{~km} \mathrm{~s}^{-1}$ (see Table 1 in Paper XI). Presently we do not know the distributions of the rotational velocities at these non-solar metallicities and thus we do not know if the adopted initial velocity corresponds to the average observed values. It may be that at lower metallicities, the initial velocity distribution contains a larger number of high initial velocities (Maeder et al. 1999), in which case the effects of rotation described below would be underestimated at low metallicity.

- The $\mathrm{Ne}-\mathrm{Na}$ and $\mathrm{Mg}-\mathrm{Al}$ nuclear reaction chains have been included in addition to the usual nuclear reactions for the $\mathrm{H}$ and He-burning phases. The long-lived $\left(t_{1 / 2}=7.05 \times\right.$ $\left.10^{5} \mathrm{yr}\right){ }^{26} \mathrm{Al}^{g}$ ground state is considered as a separate species from its short-lived $\left(t_{1 / 2}=6.35 \mathrm{~s}\right){ }^{26} \mathrm{Al}^{m}$ isomeric state. In the following, for purpose of simplicity, the longlived ${ }^{26} \mathrm{Al}^{g}$ ground state will be denoted by the symbol ${ }^{26} \mathrm{Al}$. The nuclear reaction rates are taken from the NACRE compilation (Angulo et al. 1999). Arnould et al. (1999) have studied in a simple parametric model the uncertainties on the abundances of some elements due to the uncertainties of the nuclear reaction rates. For what concerns ${ }^{26} \mathrm{Al}$ they reach the interesting conclusion that the large uncertainties on the ${ }^{26} \mathrm{Al}(p, \gamma)^{27} \mathrm{Si}$ reaction rate scarcely affect the abundance of ${ }^{26} \mathrm{Al}$, considering that "even the highest NACRE proton capture rates are not fast enough for leading to a substantial destruction of the two Al isotopes by the time $H$ is consumed" (Arnould et al. 1999).

Let us finally recall that the wind anisotropies induced by rotation were neglected. This last choice appears justified in view of the results obtained in Paper X. Indeed for the initial velocities considered $\left(v_{\text {ini }}=300 \mathrm{~km} \mathrm{~s}^{-1}\right)$, the effects of the wind anisotropies have been shown to be very small. Let us however emphasize that this is not true for higher initial velocities (Maeder 2002).

\section{Effects of rotation on ${ }^{26} \mathrm{Al}$ production by WR stars}

${ }^{26} \mathrm{Al}$ is mainly produced by proton capture on ${ }^{25} \mathrm{Mg}$ seeds in the convective core during the central Hydrogen Burning phase (see Figs. 1 and 2). In non-rotating stars, part of the ${ }^{26} \mathrm{Al}$ produced in the core, depending on the initial stellar mass, may appear at the surface due to the removal of the envelope by 


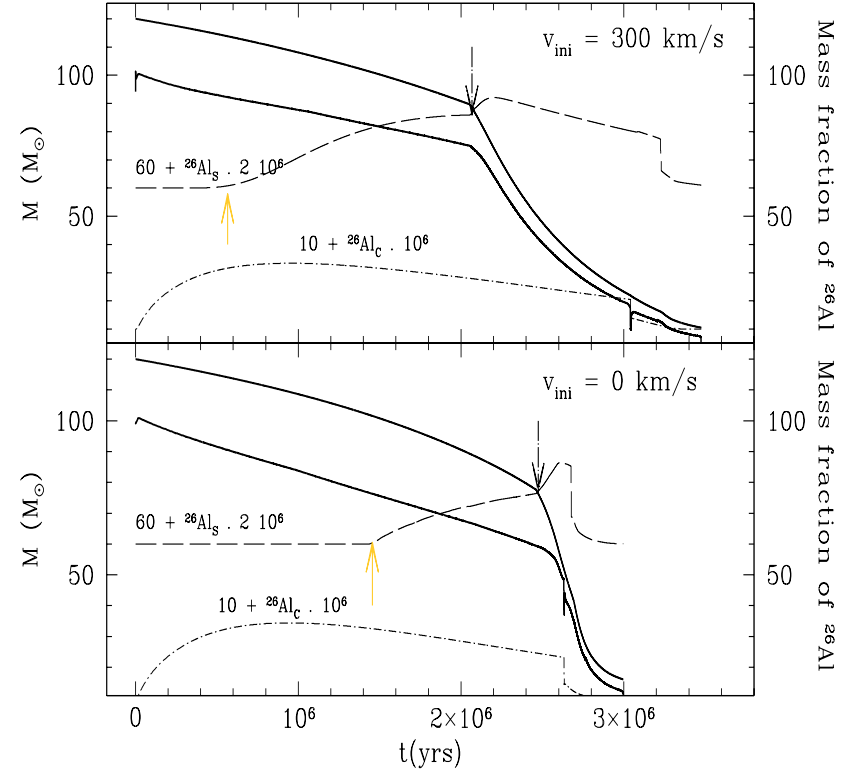

Fig. 1. Evolution as a function of time of surface $\left({ }^{26} \mathrm{Al} \mathrm{s}_{\mathrm{s}}\right)$ and central $\left({ }^{26} \mathrm{Al}_{\mathrm{c}}\right)$ mass fraction of ${ }^{26} \mathrm{Al}$ for both rotating (upper panel) and nonrotating (lower panel) $120 M_{\odot}$ stellar models at $Z=0.020$. Light dashed lines show the scaled values of the mass fractions $60+{ }^{26} \mathrm{Al}_{\mathrm{s}} \times$ $2 \times 10^{6}$ and $10+{ }^{26} \mathrm{Al}_{\mathrm{c}} \times 10^{6}$. Bold solid lines mimic a Kippenhahn like diagram, and show the evolution of the mass of the convective core and that of the total stellar mass as a function of time. The downgoing arrows indicate the beginning of the strong wind phase, and the up-going arrows indicate the beginning of the surface ${ }^{26} \mathrm{Al}$ enrichment.

stellar winds. From this point on, stellar winds enrich the interstellar medium in ${ }^{26} \mathrm{Al}$. Including rotation somewhat modifies this picture, as we will show in the following sections.

Before entering into the details of the effects of rotation on ${ }^{26} \mathrm{Al}$ release by WR stars, let us briefly recall those effects of rotation on massive star evolution which may be relevant in the context of ${ }^{26} \mathrm{Al}$ nucleosynthesis:

1. Rotation allows diffusion of the chemical species from the convective core to the radiative envelope and vice versa; this results in changes of the surface abundances already during the Main Sequence phase. The surface enrichments given by the models are generally in good agreement with the observed ones (see Heger \& Langer 2000; Meynet \& Maeder 2000; Maeder \& Meynet 2001).

2. Rotation increases mass loss (see Sect 2). More precisely a rotating star of given initial mass and position in the HR diagram undergoes a higher mass loss than a non-rotating star (Paper VI).

3. Diffusion of elements actively contributing to the nuclear energy production inside the star together with enhanced mass loss allow massive stars to enter the WR phase at an earlier stage of their evolution. As a consequence, the WR phase lasts for longer than in the case of non-rotating stars.

4. Finally, rotation decreases the minimum initial mass for a single star to enter the WR phase (see Table 2).

These effects, when accounted for in stellar models, much improve the agreement between theory and observations for what

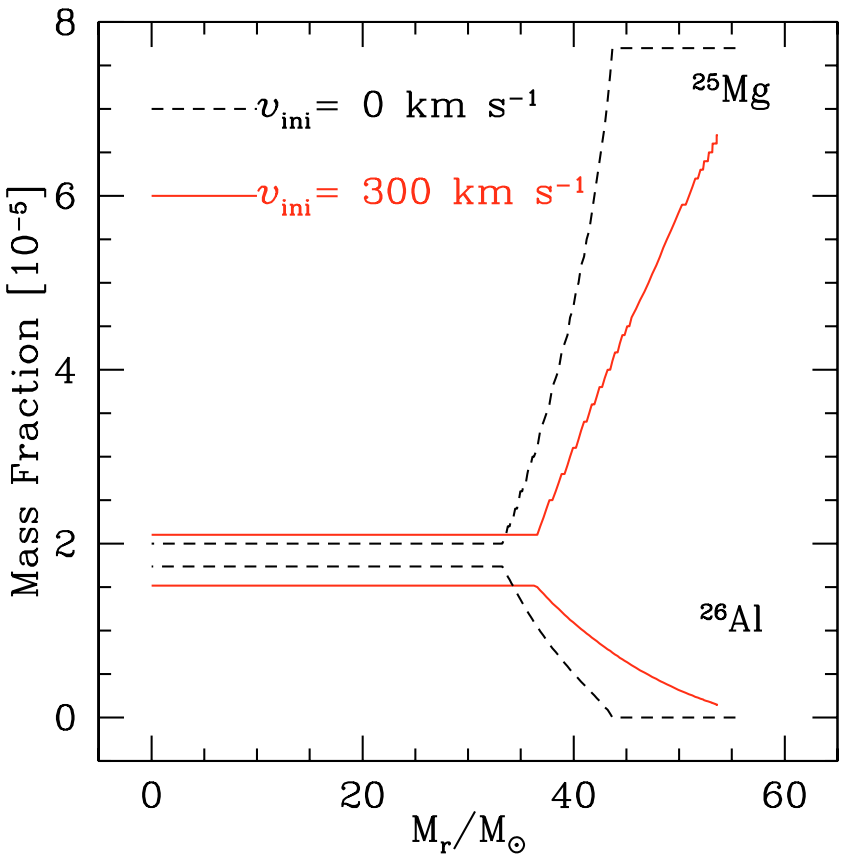

Fig. 2. Variation of the mass fractions of ${ }^{25} \mathrm{Mg}$ and ${ }^{26} \mathrm{Al}$ as a function of the lagrangian mass inside a rotating and non-rotating $60 M_{\odot}$ stellar model at $Z=0.020$ during the core $\mathrm{H}$-burning phase. In both models the mass fraction of hydrogen in the convective core is equal to 0.4 .

concerns the characteristics of the WR populations at different metallicities (see Papers X and XI). Through these different processes, rotation also modifies the quantity of ${ }^{26} \mathrm{Al}$ ejected by massive stars undergoing strong winds. In Figs. 1 to 3 , we present the evolution of the ${ }^{26} \mathrm{Al}$ abundance in test models of 60 and $120 M_{\odot}$ at different metallicities. In Fig. 4 and Table 1, we provide the wind ejected mass of ${ }^{26} \mathrm{Al}$

$M_{26}^{W}\left[M_{i}, Z_{i}\right]=\int_{0}^{\tau\left(M_{i}, Z_{i}\right)}{ }^{26} \mathrm{Al}_{S}\left(M_{i}, Z_{i}, t\right)\left|\dot{M}\left(M_{i}, Z, t\right)\right| \mathrm{d} t$

for models with $M_{i} \in\left[25 M_{\odot} ; 120 M_{\odot}\right]$ and metallicities from $Z=0.004$ to $Z=0.04$. Here we do not take the radioactive decay of ${ }^{26} \mathrm{Al}$ into account, provided that it is the production rate of ${ }^{26} \mathrm{Al}$ that is needed to estimate the contribution of WR stars to the present galactic mass of ${ }^{26} \mathrm{Al}$.

The effects of rotation on ${ }^{26} \mathrm{Al}$ nucleosynthesis are the followings:

1. As can be seen from Fig. 1, rotation slows down the decrease in mass of the convective core during central hydrogen burning phase. This results from the fact that rotational mixing continuously brings fresh $\mathrm{H}$-fuel into the core. The regions where ${ }^{26} \mathrm{Al}$ is synthesized are closer to the surface, making it easier for the mixing to connect both regions, thus favouring an increase of the quantity of ${ }^{26} \mathrm{Al}$ ejected. One notes also from Fig. 1 that whether rotation is taken into account or not, the maximum content of ${ }^{26} \mathrm{Al}$ inside the convective core is about the same in both cases, and it is reached at $1 \mathrm{Myr}$. Afterwards, the amount of ${ }^{26} \mathrm{Al}$ slowly decreases as its production via the reaction ${ }^{25} \mathrm{Mg}(p, \gamma){ }^{26} \mathrm{Al}$ is not sufficient anymore to compensate its reduction due to radioactive decay. 
Table 1. Wind ejected mass of ${ }^{26} \mathrm{Al}$ in units of $10^{-4} M_{\odot}$ for both rotating and non-rotating models at the different metallicities considered. Bold values are for models computed with metallicity dependent mass loss during the WR phase.

\begin{tabular}{c|c|cccccc}
\hline \hline$Z$ & $v_{\text {ini }}\left(\mathrm{km} \mathrm{s}^{-1}\right)$ & $M_{i}=120 M_{\odot}$ & $M_{i}=85 M_{\odot}$ & $M_{i}=60 M_{\odot}$ & $M_{i}=40 M_{\odot}$ & $M_{i}=30 M_{\odot}$ & $M_{i}=25 M_{\odot}$ \\
\hline \multirow{3}{*}{0.04} & 300 & 25.74 & 13.07 & 7.567 & 1.980 & & 0.962 \\
& 300 & $\mathbf{2 2 . 4 4}$ & $\mathbf{1 1 . 8 7}$ & $\mathbf{7 . 2 0 5 6}$ & $\mathbf{2 . 0 2 9}$ & & 0.029 \\
\hline 0.02 & 0 & 21.62 & & 3.021 & & 0.345 \\
& 0 & 12.29 & 5.83 & 2.181 & & 0.001 \\
\hline 0.008 & 300 & 2.566 & & 1.304 & & 0.096 & \\
\hline 0.004 & 300 & 0.701 & & 0.297 & 0.210 & 0.002 & \\
\hline
\end{tabular}

2. Due to rotational mixing, the ${ }^{26} \mathrm{Al}$ surface abundance begins to change at a much earlier time in the rotating model. This can also be seen from Fig. 2 which shows the variations inside rotating and non-rotating $60 M_{\odot}$ stellar models of the mass fractions of ${ }^{25} \mathrm{Mg}$ and ${ }^{26} \mathrm{Al}$ at the middle of the core H-burning phase. While the abundances of these two elements are very similar, the main differences arise in the radiative envelope: in the non-rotating model, above the transition zone left over by the recession of the convective core, the abundances present a flat profile at a level corresponding to the initial abundance (which is of course 0 for ${ }^{26} \mathrm{Al}$ ). Instead in the rotating model, the inwards diffusion of ${ }^{25} \mathrm{Mg}$ and the outwards diffusion of ${ }^{26} \mathrm{Al}$ smooth the profiles and produce changes of the surface abundances while mass loss has not yet uncovered layers previously pertaining to the convective core.

3. From Figs. 1 and 3, it appears that most of the ${ }^{26} \mathrm{Al}$ is ejected while the star is at the beginning of the WR phase. Indeed at this stage, the layers removed by the stellar winds are heavily loaded in ${ }^{26} \mathrm{Al}$ and the mass loss rate is high. When layers processed by He-burning appear at the surface, the quantity of ${ }^{26} \mathrm{Al}$ much decreases, because ${ }^{26} \mathrm{Al}$ is destroyed (mainly by neutron capture) at the beginning of the core He-burning phase. Since rotating stars enter the WR phase at an earlier evolutionary stage than non-rotating ones (see Fig. 1), the period during which ${ }^{26} \mathrm{Al}$ is released into the ISM is longer, and the net yield of this nuclide is also increased when rotation is taken into account. This appears clearly in Table 1, where we show the total wind ejected mass of ${ }^{26} \mathrm{Al}$ for the computed models.

4. The lowering of the minimum mass for a star to enter the WR phase contributes to enhance considerably the total contribution of WR stars to the global galactic content of ${ }^{26} \mathrm{Al}$. Less massive stars, favoured by the IMF, will now contribute to the ${ }^{26} \mathrm{Al}$ enrichment of the medium through their winds, whereas their non-rotating counterparts could only contribute through the supernova explosion (see Sect. 4).

\subsection{Relative effects of mass loss and rotation on ${ }^{26} \mathrm{Al}$}

Values listed in Table 1 show that for the higher metallicity models the ${ }^{26} \mathrm{Al}$ mass ejected is increased by a factor of about 2.5 for typical WR progenitors with $M_{\text {init }} \sim 40-85 M_{\odot}$.
For the $25 M_{\odot}$ model, the ${ }^{26} \mathrm{Al}$ mass released by rotating models is 33 times higher than the one obtained for non-rotating ones, mainly because in the latter case, these stars do not enter the WR phase and contribute very few through their winds to the ${ }^{26} \mathrm{Al}$ enrichment of the interstellar medium. In the lower mass stars, the wind during the WR phase is weaker than for the more massive ones, and rotation-induced mixing is the main mechanism driving the evolution of the surface abundance patterns. On the contrary, in the mass range $] 60 M_{\odot} ; 120 M_{\odot}$ ], the surface abundances during the WR phase essentially result from the efficiency of the wind to peel off the star, so that the effects of rotation-induced mixing do not appear that strikingly when comparing rotating and non-rotating models (see also Fig. 4).

It is interesting to note from Fig. 4 that the yields from Meynet et al. (1997), computed for non-rotating models with enhanced mass loss are lower than the ones obtained for rotating models with lower mass loss rates compatible with the most recent observational determination (see Sect. 2). This again points out the importance of rotation, non solely in increasing mass loss, but mainly in allowing for transport of the chemicals throughout the stellar interiors.

\subsection{Metallicity dependence of ${ }^{26} \mathrm{Al}$ yields}

As shown in previous papers (Meynet et al. 1997; Vuissoz et al. 2004), the amount of ${ }^{26} \mathrm{Al}$ both synthesized and expelled by a WR star increases with metallicity. This is already the case for non-rotating models, and rotation adds to this effect as can be seen in Figs. 3 and 4.

In Fig. 3, we present the evolution of the surface and center mass fraction of ${ }^{26} \mathrm{Al}$ as a function of time for rotating $120 M_{\odot}$ stellar models at various metallicities. One sees that the higher the metallicity, the larger the amount of ${ }^{26} \mathrm{Al}$ produced in the convective core and the earlier ${ }^{26} \mathrm{Al}$ appears at the surface. Thus for a given initial mass and rotation, the mass of ${ }^{26} \mathrm{Al}$ ejected is greater at higher metallicity. This is also well illustrated in Fig. 4. From the present models one expects a relation of the type $Y\left({ }^{26} \mathrm{Al}, M_{\text {ini }}\right) \propto Z_{\text {ini }}^{\beta}$ between the yield and the metallicity. Here, the value of $\beta$ depends on both initial mass and rotation. Typically from the rotating stellar models with $M_{\mathrm{ini}}=40,60$ and $120 M_{\odot}$, one obtains $\beta=1.4,1.8$ and 1.4 respectively in the metallicity range from $Z=0.008$ to 0.040 . For the nonrotating 60 and $120 M_{\odot}$ and in the metallicity range from 0.02 


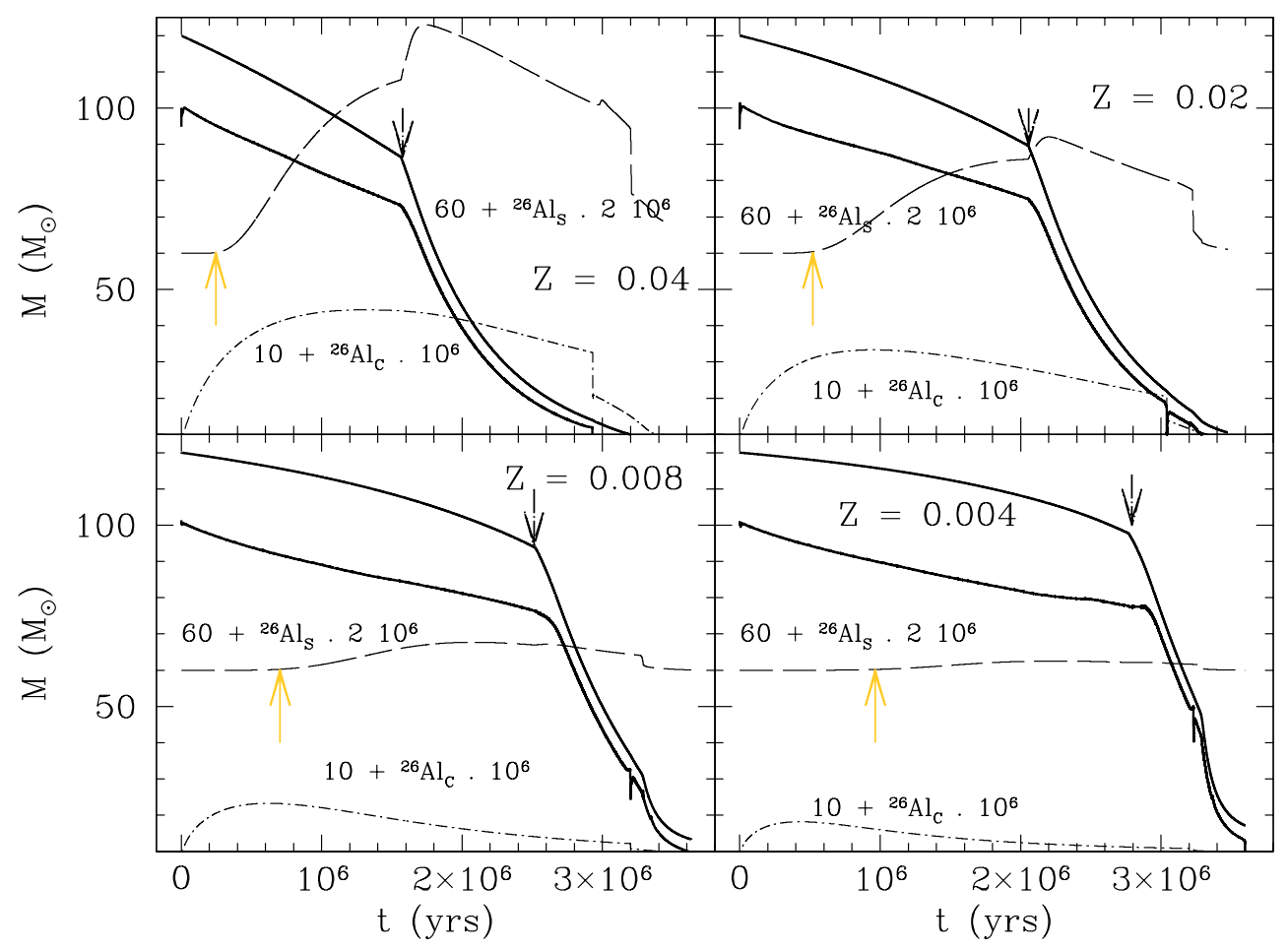

Fig. 3. Same as Fig. 1 for rotating models of a $120 M_{\odot}$ star at four different metallicities, as indicated on the plots.

to 0.04 , one obtains values of $\beta$ equal to 1.2 and 1.9 respectively. As can be seen from these numerical examples, the concomitant effects of rotation and mass loss are intricate and it is difficult to draw more general trends for what concerns the sensitivity of the yields on the initial metallicity.

\subsection{Effects of metallicity dependent mass loss during the WR phase}

For twice the solar metallicity, we computed models using Crowther et al. (2002) prescription for the mass loss rates during the WR phase. For this metallicity, the derived mass loss rates are multiplied by a factor $\sim 1.4$. The resulting wind ejected masses of ${ }^{26} \mathrm{Al}$ are listed in bold face in Table 1 . For the most massive stars, the masses of ejected ${ }^{26} \mathrm{Al}$ do not differ by more than $13 \%$ when the metallicity dependent mass loss rates are used. At first sight this may appear surprising. However the most massive stars enter the WR phase while central H-burning is still going on. In that case a stronger mass loss produces a more important reduction of the convective H-burning core, accompanied by a severe reduction of the central temperature. The end of the H-burning phase is then lengthened, and thus the ${ }^{26} \mathrm{Al}$ present inside the convective core has more time to decay in situ before being expelled.

The changes are however small and therefore one can conclude that the use of metallicity dependent mass loss rate does not affect significantly the results.

\subsection{Yields dependence on initial velocity}

Finally let us recall that in Table 1 , the yields in ${ }^{26} \mathrm{Al}$ for rotating models are given for an initial rotation velocity on the
ZAMS of $300 \mathrm{~km} \mathrm{~s}^{-1}$. This value corresponds to an average velocity during the MS phase between 200 and $250 \mathrm{~km} \mathrm{~s}^{-1}$, i.e. well in the observed velocity range for the OB Main Sequence stars at solar metallicity. In order to estimate the effects of a higher initial velocity, we have computed, for solar metallicity, a $60 M_{\odot}$ stellar model with $v_{\text {ini }}=500 \mathrm{~km} \mathrm{~s}^{-1}$. The quantity of ${ }^{26} \mathrm{Al}$ ejected by the stellar winds is in this case of $2.6 \times 10^{-4} M_{\odot}$, which is less than $20 \%$ higher than the yields obtained from the model with $v_{\text {ini }}=300 \mathrm{~km} \mathrm{~s}^{-1}$. Thus at least in this particular case, the yields do not appear to be very sensitive to a change of the initial velocity in the range between 300 and $500 \mathrm{~km} \mathrm{~s}^{-1}$.

\section{Contribution of WR stars to the ${ }^{26} \mathrm{Al}$ galactic content}

Let us first consider a simple way to estimate the global contribution of WR stars to the present day ${ }^{26} \mathrm{Al}$ content of the Galaxy. According to van der Hucht (2002), the galactic distribution of WR stars shows a projected surface density of 2.7 WR stars per $\mathrm{kpc}^{2}$ in the solar neighbourhood. Extrapolating this estimate of the surface density to the whole Galaxy gives a total number of WR stars in the Galaxy of the order of about 2000. Since the average lifetime of WR stars is $\sim 1 \mathrm{Myr}$ (e.g. Paper XI) this gives a galactic frequency $f_{\mathrm{WR}} \sim$ $2000 \mathrm{Myr}^{-1}$. Let us note that these numbers are likely to be lower limits, since the star formation rate and the number of WR to O-type stars is much higher in inner galactic regions.

The galactic frequency of WR stars may also be estimated by using a stellar Initial Mass Function (IMF) and the current death rate of massive stars in the Milky Way. Observations of external spiral galaxies indicate that the expected supernova 
Table 2. Minimum mass $M_{\mathrm{LWR}}$ for a star to enter the WR phase as a function of $Z$ and $v_{\text {ini }}$ (from Meynet \& Maeder 2005).

\begin{tabular}{c|c|cccc}
\hline \hline & $\begin{array}{c}v_{\text {ini }} \\
\left(\mathrm{km} \mathrm{s}^{-1}\right)\end{array}$ & $\begin{array}{c}Z \\
0.04\end{array}$ & $\begin{array}{c}Z \\
0.02\end{array}$ & $\begin{array}{c}Z \\
0.008\end{array}$ & $\begin{array}{c}Z \\
0.004\end{array}$ \\
\hline$M_{\mathrm{LWR}}$ & 0 & 29 & 37 & 42 & 52 \\
$\left(M_{\odot}\right)$ & 300 & 21 & 22 & 25 & 32 \\
\hline
\end{tabular}

frequency in our Galaxy is $f_{\mathrm{SN}}=2.5_{-0.5}^{+0.8}$ per century, with $85 \%$ of them coming from massive stars (Tammann et al. 1994). We adopt then $f_{\mathrm{SN}}=2$ per century for the death rate of massive stars in the Milky Way. We use a Salpeter (1955) stel$\operatorname{lar} \mathrm{IMF}^{3}, \Phi(M) \propto M^{-(1+x)}$ with $x=1.35$. The WR frequency is then given by:

$f_{\mathrm{WR}}=f_{\mathrm{SN}} \frac{\int_{M_{\mathrm{LWR}}}^{M_{\mathrm{UP}}} \Phi(M) \mathrm{d} M}{\int_{M_{\mathrm{LSN}}}^{M_{\mathrm{UP}}} \Phi(M) \mathrm{d} M} \sim f_{\mathrm{SN}}\left(\frac{M_{\mathrm{LWR}}}{M_{\mathrm{LSN}}}\right)^{-x}$,

where $M_{\mathrm{LSN}}$ and $M_{\mathrm{LWR}}$ are the lowest masses of stars that may become supernovae and WR, respectively. The former is $M_{\mathrm{LSN}} \sim 8 M_{\odot}$, while the latter depends on metallicity and adopted mass loss rates. According to our calculations with rotation $M_{\mathrm{LWR}} \sim 21 M_{\odot}$ for $Z=2 Z_{\odot}$ (see Table 2 ). We consider here the high metallicity models since, most of the WR stars are found in the inner regions of our Galaxy where the metallicity is higher (see below). Taking these values into account one obtains $f_{\mathrm{WR}} \sim 0.2 f_{\mathrm{SN}}$, i.e. a WR frequency of 0.4 per century or $4000 \mathrm{WR}$ stars per Myr. On the other hand, from the yields of Table 1 and our adopted IMF, an average WR yield of $\left\langle M_{26}^{W}\right\rangle \sim 3.4 \times 10^{-4} M_{\odot}$ can be defined for $Z=2 Z_{\odot}$ stars.

The collective production rate of ${ }^{26} \mathrm{Al}$ in the Galaxy by WR stars can then be expressed as

$\dot{M}_{26} \sim 1.4 \frac{\left\langle M_{26}^{W}\right\rangle}{3.4 \times 10^{-4}} \frac{f_{\mathrm{WR}}}{4000} \quad M_{\odot} \mathrm{Myr}^{-1}$.

In the case of a stationary regime as it assumed to be the case in the Milky Way, the galactic mass of ${ }^{26} \mathrm{Al}$ does not vary with time, so that at a given time $t$, the production $\left(P_{26}(t)\right)$ and destruction $\left(D_{26}(t)\right)$ rates of ${ }^{26} \mathrm{Al}$ are equal. The destruction rate is easy to evaluate, considering the evolution of the ${ }^{26} \mathrm{Al}$ mass solely due to its decay, e.g.

$M_{26}(t)=M_{26}\left(t_{0}\right) \cdot \mathrm{e}^{-\frac{t}{\tau}} \Rightarrow D_{26}=\frac{M_{26}}{\tau}$.

As $P_{26}(t)=D_{26}(t)$, the production rate of ${ }^{26} \mathrm{Al}$ is then given by:

$P_{26}^{\mathrm{gal}}=D_{26}^{\mathrm{gal}}=\dot{M}_{26}^{\mathrm{gal}}=\frac{M_{26}^{\mathrm{gal}}}{\tau} M_{\odot}$ per unit time.

The total mass of ${ }^{26} \mathrm{Al}$ originating from WR stars present in the Galaxy is related to the production rate of ${ }^{26} \mathrm{Al}$ by these stars

\footnotetext{
${ }^{3}$ In the following, we will also discuss the effect of using a larger slope $(x=1.7)$ that could be more appropriate for massive stars when accounting for binarity corrections (Scalo 1986; Kroupa et al. 1993).
}

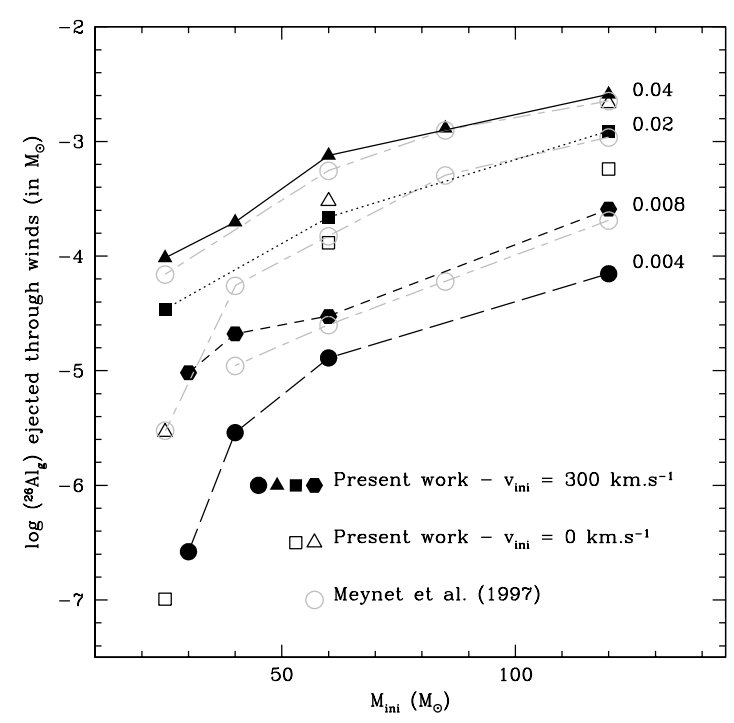

Fig. 4. ${ }^{26} \mathrm{Al}$ yields, as listed in Table 1, as a function of initial stellar mass and metallicity. Filled symbols are for rotating models with $v_{\text {ini }}=300 \mathrm{~km} \mathrm{~s}^{-1}$, open black squares and triangles are for nonrotating models and open grey circles connected by long-short dashed lines are from Meynet et al. (1997). The corresponding metallicity is indicated on the right part of the figure.

through Eq. (4). The mean lifetime of ${ }^{26} \mathrm{Al}$ being of the order of $1.04 \mathrm{Myr}$, we finally have:

$M_{26}^{\text {gal }}=\tau_{26} \times \dot{M}_{26}^{\text {gal }} \approx \dot{M}_{26}^{\text {gal }} M_{\odot}$.

Thus Eq. (3) (and Eq. (6) in the following) in addition to give the injection rate of ${ }^{26} \mathrm{Al}$ by WR stars, can also be used to evaluate the total mass of ${ }^{26} \mathrm{Al}$ originating from WR stars. According to our rough estimate from Eq. (3), the mass of ${ }^{26} \mathrm{Al}$ contributed by WR stars amounts to $45 \%$ and even $70 \%$ of the observationally inferred mass of about $1.8 M_{\odot}$ (Knödlseder 1997).

In a more formal way, the ${ }^{26} \mathrm{Al}$ production rate (in solar mass per unit time) from galactic WR stars may be evaluated as

$\dot{M}_{26}=\int_{0}^{\mathrm{R}} 2 \pi r \sigma(r) \int_{M_{\mathrm{LWR}}(r)}^{120} \Phi(M) M_{26}^{W}\left[M_{i}, Z_{i}(r)\right] \mathrm{d} M \mathrm{~d} r$,

where $\sigma(r)$ is the Star Formation Rate (hereafter SFR) per unit surface in the Galaxy given as a function of galactocentric distance $r$ and $M_{26}^{W}$ is the mass of ${ }^{26} \mathrm{Al}$ ejected by a star of initial mass $M_{i}$ and initial metallicity $Z_{i}(r)$, according to Eq. (1).

To account for the metallicity dependence of the ${ }^{26} \mathrm{Al}$ yields and of the lowest WR mass $M_{\mathrm{LWR}}$ in Eq. (6), we use the $Z$-dependent values given in Tables 1 and 2 respectively.

The integration in Eq. (6) requires the following ingredients:

- Normalization of both the SFR $\sigma(r)$ and the IMF $\Phi(M)$. We can either rely, as in our first simple estimate, on an approximate frequency of SNae events in the Galaxy; in this case, the integral $\int_{0}^{\mathrm{R}} 2 \pi r \sigma(r) \mathrm{d} r \int_{8}^{120} \Phi(M) \mathrm{d} M$ is normalized to $2 \times 10^{4} \mathrm{Myr}^{-1}$ ( 2 cc-SNae per century in the Galaxy). 
We can also follow Knödlseder (1999), and normalize the SFR so that the total predicted Lyman continuum photon production of the Galactic population defined by the IMF matches the observed Lyman continuum luminosity $Q$. The actual value of $Q$ as deduced by McKee \& Williams (1997) is equal to $2.56 \times 10^{53}$ photons s ${ }^{-1}$. In that case, according to Eqs. (18) and (20) of Knödlseder (1999), we will have $\int_{1}^{120} \Phi(M) \mathrm{d} M=1$ and the star formation rate $\int_{0}^{\mathrm{R}} 2 \pi r \sigma(r) \mathrm{d} r$ normalized to $\frac{Q}{\int_{8}^{120} s_{\mathrm{H}}(M) \Phi(M) \tau_{\text {total }}(M) \mathrm{d} M}, s_{\mathrm{H}}(M)$ being the timeaveraged Lyman continuum luminosity and $\tau_{\text {total }}(M)$ the total lifetime of a star of initial mass $M$. To evaluate these two quantities, we make use of Eq. (21) of Knödlseder (1999), which is a rough approximation when taking rotation into account, considering that these estimates are for non-rotating models with different mass loss prescriptions than the ones used in the present paper. Keeping this in mind, we nevertheless found it interesting to estimate the effect of normalization on the ${ }^{26} \mathrm{Al}$ injection rates predicted by Eq. (6), and used both SNae and Lyman continuum for this purpose.

- The metallicity gradient plays an important role for determining the global contribution of WR stars to the galactic ${ }^{26} \mathrm{Al}$ enrichment. In order to illustrate quantitatively this point, we performed three estimates with three different galactic metallicity distributions in the Galaxy.

Our first choice (model Z1) is based on the metallicity gradient deduced from observations of HII regions by Shaver et al. (1983) and on the galactic centre metal abundance obtained by Najarro (1999). It corresponds to a metallicity gradient of $\mathrm{d} \log Z / \mathrm{d} r=-0.07 \mathrm{dex} \mathrm{kpc}^{-1}$ (with $Z(8.5 \mathrm{kpc})=0.02)$ in the whole region lying between 1.7 and $15 \mathrm{kpc}$. In the inner $1.7 \mathrm{kpc}$, the metallicity was taken equal to 0.06 (i.e. three times solar). Since we did not compute models at such a high metallicity, we estimated the ${ }^{26} \mathrm{Al}$ yields for metallicities above 0.04 extrapolating those obtained at solar and twice the solar metallicity. Our second choice (model Z2) is based on the metallicity gradient proposed by Andrievsky et al. (2004), which relies on cepheids observation. They divide the Galaxy into three zones: Zone I encompasses the region between 4.0 and $6.6 \mathrm{kpc}$. In this zone metallicity varies according to the $\operatorname{law} \log Z / Z_{\odot}=-0.128 R_{\mathrm{G}}+0.930, R_{\mathrm{G}}$ being the galactocentric distance expressed in kpc. Zone II extends from 6.6 to $10.6 \mathrm{kpc}$ and has a metallicity $\operatorname{law} \log Z / Z_{\odot}=$ $-0.044 R_{\mathrm{G}}+0.363$ (for $R_{\mathrm{G}}=8.5 \mathrm{kpc}, Z \sim Z_{\odot}$ ). The third zone goes from 10.6 to $14.6 \mathrm{kpc}$ and has a metallicity law of the form ${ }^{4} \log (Z+0.024) / Z_{\odot}=+0.004 R_{\mathrm{G}}+0.256$. For galactocentric distances inferior to $4 \mathrm{kpc}$, we take a constant value of the metallicity equal to $2.62 \times Z_{\odot}$.

Finally in model $Z 3$, we consider a much shallower gradient of $\mathrm{d} \log Z / \mathrm{d} r=-0.04 \mathrm{dex} \mathrm{kpc}^{-1}$ in the whole region between 4.4 and $12.9 \mathrm{kpc}$ as suggested by Daflon \& Cunha (2003). For galactocentric distances below 4.4, we

\footnotetext{
${ }^{4}$ There is an error in the expression given by Andrievsky et al. (2004) for this zone, that can be corrected considering $\log (Z+$ $0.024) / Z_{\odot}$ instead of $\log Z / Z_{\odot}$.
}

suppose a constant metallicity equal to 0.03 and for galactocentric distance above $12.9 \mathrm{kpc}$, we take a constant metallicity equal to 0.013 .

These three hypotheses not only account for the various values of the metallicity gradients found in the literature, but also for the different views on the metallicity of the galactic centre. For instance Ramírez et al. (2000) and Najarro et al. (2004) find that the mean $[\mathrm{Fe} / \mathrm{H}]$ of the Galactic Centre stars is similar to that of the solar neighbourhood stars, while Najarro (1999) obtains values between 2 and 3 times the solar metallicity.

- Different estimates can be found in the literature for the radial distribution of the present Star Formation Rate in the Galaxy $\sigma(r)$. We chose to consider two different prescriptions: Paladini et al. (2004), give the distribution of HII regions in our Galaxy as a function of the galactocentric distance. HII regions are considered to be good tracers of the SFR, and we suppose that their surface density distribution is directly proportional to it. Wang \& Silk (1994), on an other hand, give the SFR per unit surface, based on observations of various tracers (Lyman continuum photons from HII regions; pulsars; supernova remnants) and normalized to the solar neighbourhood.

Applying Eq. (6) with a Salpeter IMF slope, a SFR from Wang \& Silk (1994) and a normalization on the rate of SNae events, leads to an injection rate of $\dot{M} \sim 0.3-0.6 M_{\odot} / \mathrm{Myr}$ of ${ }^{26} \mathrm{Al}$ for the non-rotating models and to values between 0.9 and $1.3 M_{\odot} / \mathrm{Myr}$ for the rotating models, not far from the order of magnitude estimates performed above (see Eq. (3)). Rotation, all other physical ingredients being the same, thus roughly doubles the contribution of the WR stellar winds to the galactic ${ }^{26} \mathrm{Al}$ enrichment, as can be seen from values in Table 3 . This remark holds whatever the combination of the physical ingredients entering Eq. (6) used. In that respect, rotation (for the adopted value for the initial velocity, $v_{\text {ini }}=300 \mathrm{~km} \mathrm{~s}^{-1}$ ) has a similar impact on the global contribution of the WR stars as an enhancement of mass loss rates (see Meynet et al. 1997).

In Figs. 5-8, we compare the theoretical mass surface density profiles of ${ }^{26} \mathrm{Al}, \Sigma_{26}$ (dashed histograms) with the profile deduced from COMPTEL data analysis (Knödlseder 1997; black curve). In Fig. 5, we use the SFR from Wang \& Silk (1994), normalized to a rate of 2 SNae per century. In Figs. 6 and 7, the SFR is estimated following Fig. 3 of Paladini et al. (2004), and it is normalized either on the SNae rate (Fig. 6), or on the total galactic Lyman continuum luminosity (Fig. 7). For each case we compare the effect of rotation and of the adopted galactic metallicity gradient. In Figs. 5 to 7, we used a Salpeter IMF; Fig. 8 is analogous to Fig. 6 (right panels) and Fig. 7 (right panels) but for a Kroupa IMF $\left(\Phi(M) \propto M^{-2.7}\right)$.

- The theoretical predictions are in general below the observed surface density curve. This is expected since in our estimate we only account for the contribution of the winds of WR stars, while other sources as the supernovae likely also contribute.

- In the present theoretical framework, the fraction contributed by other sources could be estimated from the 
Table 3. ${ }^{26} \mathrm{Al}$ injection rates predicted by the different models computed: (A) Salpeter's IMF, SFR from Wang \& Silk (1994), SNae normalization (Fig. 5); (B) Salpeter's IMF, SFR from Paladini et al. (2004), SNae normalization (Fig. 6); (C) Salpeter's IMF, SFR from Paladini et al. (2004), Lyc normalization (Fig. 7); (D) Kroupa's IMF, SFR from Paladini et al. (2004), Lyc normalization (Fig. 8); (E) Kroupa's IMF, SFR from Paladini et al. (2004), SNae normalization (Fig. 8).

\begin{tabular}{c|cccccc}
\hline \hline Model & $Z 1$ & $Z 1$ & $Z 2$ & $Z 2$ & $Z 3$ & $Z 3$ \\
\hline $\begin{array}{c}v_{\text {ini }} \\
\left(\mathrm{km} \mathrm{s}^{-1}\right)\end{array}$ & 0 & 300 & 0 & 300 & 0 & 300 \\
\hline $\begin{array}{c}\dot{M}_{26}(\mathbf{A}) \\
\left(M_{\odot} \mathrm{Myr}^{-1}\right)\end{array}$ & 0.563 & 1.242 & 0.617 & 1.336 & 0.346 & 0.876 \\
\hline $\begin{array}{c}\dot{M}_{26}(\mathbf{B}) \\
\left(M_{\odot} \mathrm{Myr}^{-1}\right)\end{array}$ & 0.551 & 1.263 & 0.632 & 1.393 & 0.371 & 0.943 \\
\hline $\begin{array}{c}\dot{M}_{26}(\mathbf{C}) \\
\left(M_{\odot} \mathrm{Myr}^{-1}\right)\end{array}$ & 0.493 & 1.130 & 0.566 & 1.247 & 0.333 & 0.844 \\
\hline $\begin{array}{c}\dot{M}_{26}(\mathbf{D}) \\
\left(M_{\odot} \mathrm{Myr}^{-1}\right)\end{array}$ & 0.434 & 1.082 & 0.500 & 1.195 & 0.292 & 0.805 \\
\hline $\begin{array}{c}\dot{M}_{26}(\mathbf{E}) \\
\left(M_{\odot} \mathrm{Myr}^{-1}\right)\end{array}$ & 0.305 & 0.759 & 0.351 & 0.838 & 0.205 & 0.565 \\
\hline
\end{tabular}

discrepancy between the theoretical and observed histograms. From Figs. 5 to 7, we see that the contribution of other sources than WR stars is much larger when the yields of non-rotating models are accounted for. As recalled in Sect. 1 (see Paper XI for more details), the non-rotating models fail to fit many observed properties of WR star populations that rotating models succeed in reproducing. We will thus preferentially trust the results obtained from the rotating models.

- The higher the metallicity of the inner galactic regions $(r<6)$, the more important the WR contribution to the ${ }^{26} \mathrm{Al}$ synthesis. In particular, models labelled as Z3, with the shallow metallicity gradient and an adopted value of 0.03 for the metallicity in the innermost regions, lead to the lower ${ }^{26} \mathrm{Al}$ injection rates in all the cases presented here.

- In the outer regions, beyond $8 \mathrm{kpc}$, there is a deficit in all the models considered here when the initial rotation velocity assumed is that of $v_{\text {ini }}=300 \mathrm{~km} \mathrm{~s}^{-1}$. If the initial distribution of rotation favours faster rotation at lower metallicity, such a deficit could be smaller, but at the present time the observational evidence for such a behaviour remains indirect (Maeder et al. 1999).

- Around $50 \%$ of the total mass of ${ }^{26} \mathrm{Al}$ in the Galaxy originates from the regions between $3 \mathrm{kpc}$ and $6 \mathrm{kpc}$, whatever the metallicity gradient adopted. This is related to the shape of the SFR, which presents a peak in this region (see Fig. 3 in Prantzos \& Aubert 1995 and in Paladini et al. 2004), mainly due to the presence of a ring of molecular clouds around $5 \mathrm{kpc}$.

- The important dependence of the theoretical results on the adopted metallicity distribution in the Galaxy appears clearly through these numerical experiments. Depending

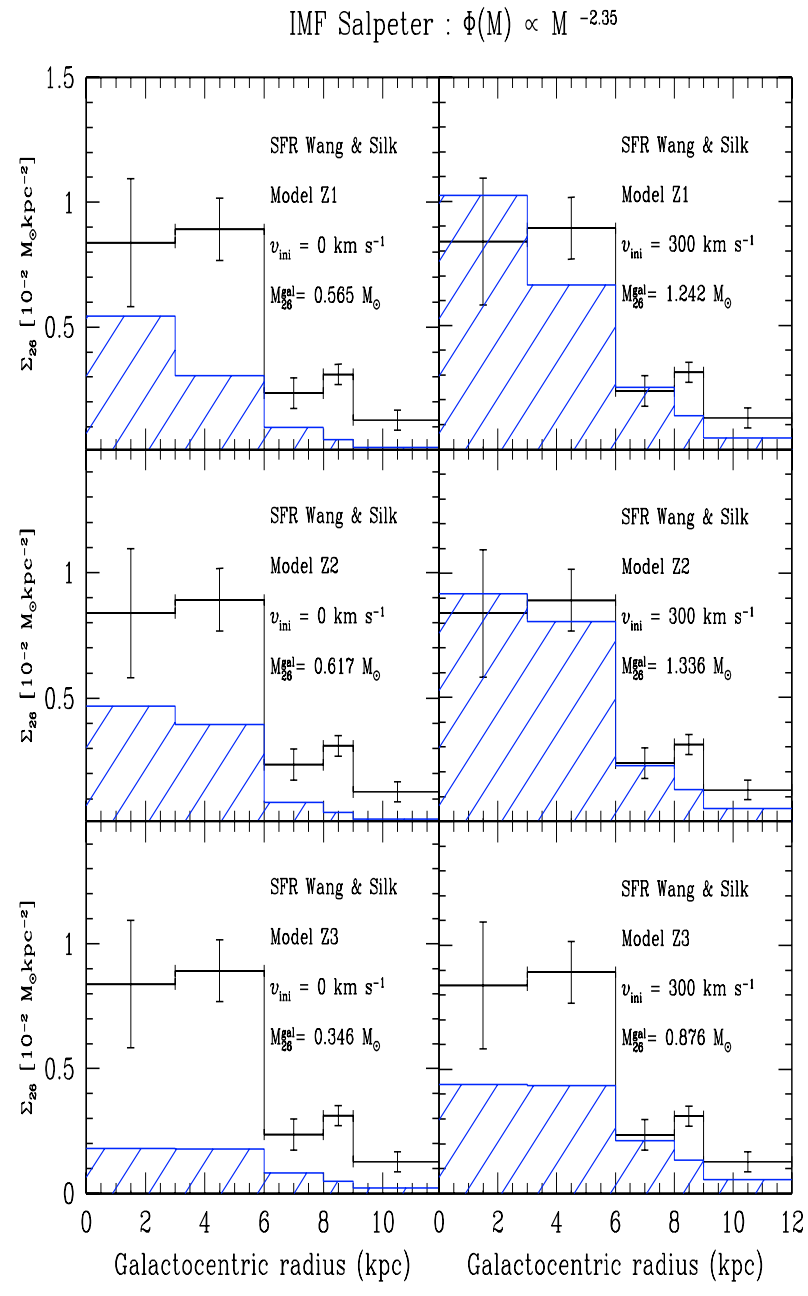

Fig. 5. Mass surface density profile of ${ }^{26} \mathrm{Al}$ (in $10^{-2} M_{\odot} \mathrm{kpc}^{-1}$ ) as a function of the galactocentric radius for an IMF index of 1.35 and a SFR from Wang \& Silk (1994), normalized in order to reproduce a rate of 2 SNae events per century in the Galaxy. The black histogram with error bars is the profile deduced from COMPTEL data analysis (Knödlseder 1997). The dashed histograms are the predicted distribution for the prescriptions adopted when using non-rotating (left panels) or rotating models (right panels).

on the metallicity distribution law adopted, both the radial distribution of the ${ }^{26} \mathrm{Al}$ and the total quantity of ${ }^{26} \mathrm{Al}$ predicted to be deposited by the WR stellar winds vary significantly. Shallower metallicity gradients, as adopted in model Z3, produce smaller values for $M_{26}^{\text {gal }}$ and smoother profiles of $\Sigma_{26}$. This conclusion holds whether rotation is included or not.

Thus from the observed distribution of $\Sigma_{26}$, it is not possible to infer constraints on the metallicity dependence of the stellar yields (even if only one source dominates), unless more reliable and concordant estimates of the galactic metallicity gradients are obtained.

- Comparing Figs. 5-7 also allows us to study the effects of the choice of the SFR or that of its normalization on the ${ }^{26} \mathrm{Al}$ distribution and injection rate by WR stars. The SFR adopted entirely determines the shape of the surface density distribution predicted: while the Wang \& Silk (1994) prescription leads to a monotonous decrease of $\Sigma_{26}$ 


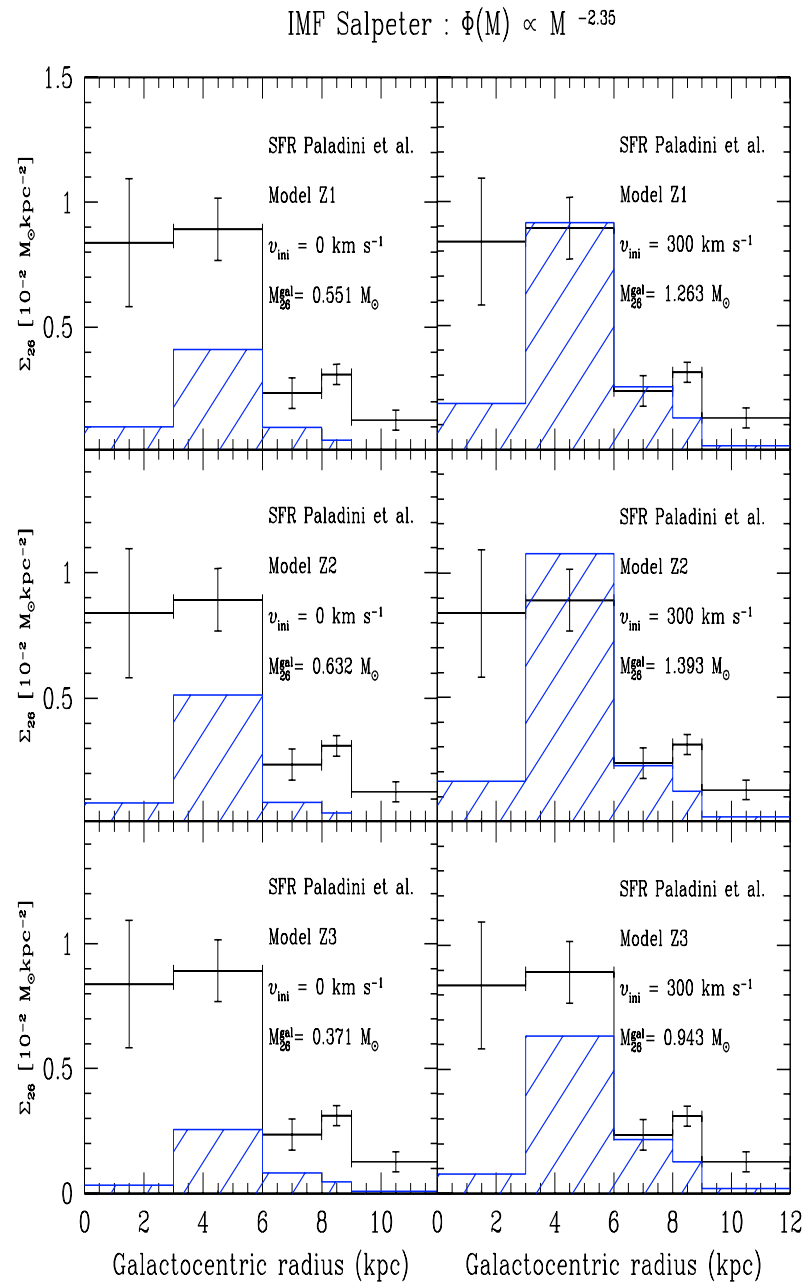

Fig. 6. Same as Fig. 5, for a SFR based on Paladini et al. (2004) number counts of HII clouds, and $\int_{0}^{\mathrm{R}} 2 \pi r \sigma(r) \mathrm{d} r \int_{8}^{120} \Phi(M) \mathrm{d} M$ normalized to $2 \times 10^{4} \mathrm{SNae} / \mathrm{Myr}$.

with increasing galactocentric radius, the values derived from Paladini et al. (2004) all present a low surface density in the inner regions, a peak in the region of the molecular clouds ring, and then a monotonous decrease, in better agreement with the nominal observed distribution ${ }^{5}$. On the other hand, as can be seen from Table 3, these differences between the two prescriptions used for the SFR (Figs. 5 and 6) only slightly affect the total contributed mass of ${ }^{26} \mathrm{Al}$ predicted, both for rotating and non-rotating WR models.

Changing the normalization, and adjusting the SFR to the match the observed galactic Lyman continuum luminosity does not change the results significantly, despite the fact that the value adopted of 2.56 photons $\mathrm{s}^{-1}$ corresponds to a lower rate of SNae events, namely 1.2 SNae per century. The masses deduced with the same value for $Q$ as Knödlseder (1999), i.e. 3.5 photons s ${ }^{-1}$ are to be multiplied by 1.37 , which leads in the most favorable case

\footnotetext{
${ }^{5}$ Note that within the errorbars from observations, the shape obtained with the Wang \& Silk (1994) prescription also matches the observed distribution.
}

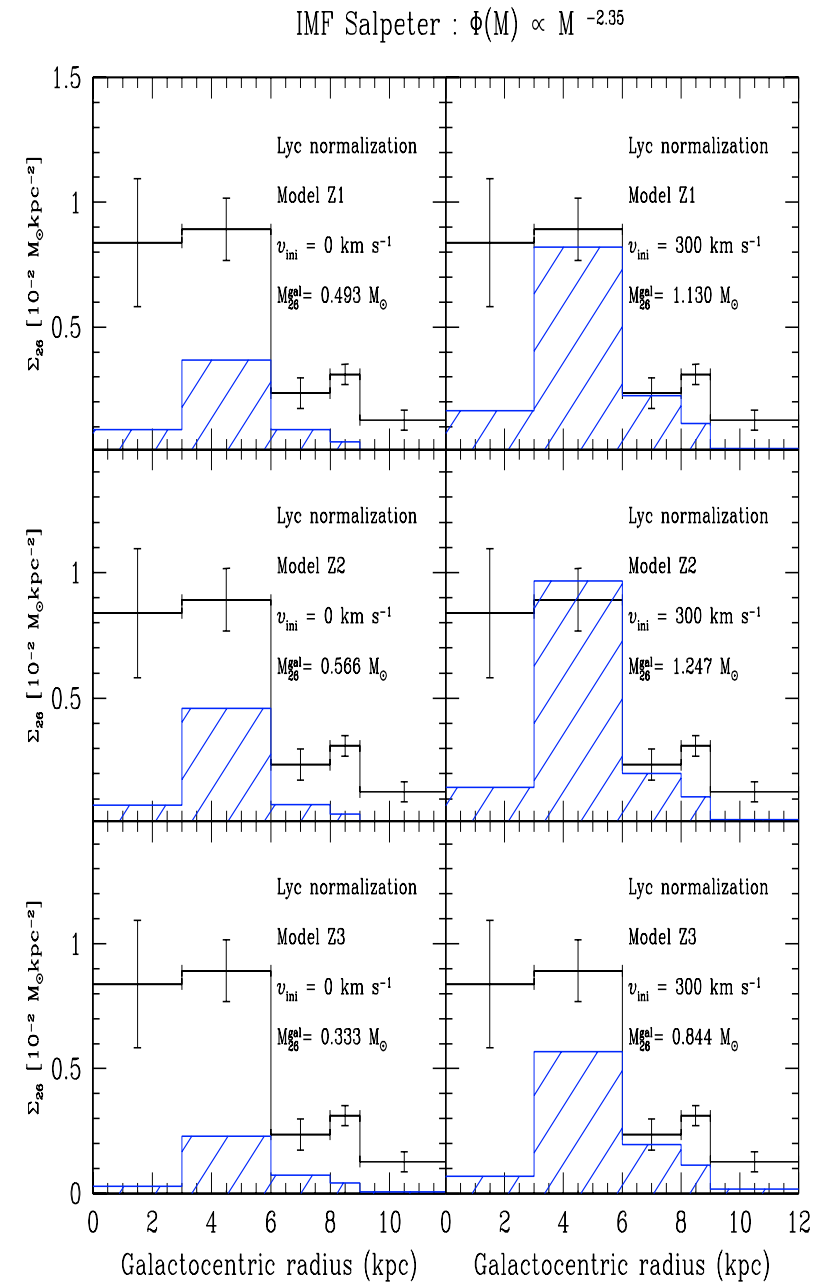

Fig. 7. Same as Fig. 5, for a SFR based on Paladini et al. (2004) number counts of HII clouds, normalized to the observed Lyman continuum luminosity of $Q=2.56 \times 10^{53}$ photons s ${ }^{-1}$.

to $1.70 M_{\odot}$ of the present galactic mass of ${ }^{26} \mathrm{Al}$ originating from WR stars.

Finally, using an IMF slope of $x=1.7$ (Scalo 1986; Kroupa 1993) rather than 1.35 lowers the total galactic mass of ${ }^{26} \mathrm{Al}$ predicted by the models as was shown in Fig. 8 and in Palacios et al. (2004 in their Fig. 3), without affecting the shape of the ${ }^{26} \mathrm{Al}$ surface density distribution.

From the above estimates and comparisons, we can conclude that when stellar models accounting for the observed properties of the WR star populations are used (i.e. rotating models), WR stars appear to be significant if not dominant contributors to the present day ${ }^{26} \mathrm{Al}$ content of the Milky Way. The predictions for the total mass of ${ }^{26} \mathrm{Al}$ originating from WR stars vary from $0.6 M_{\odot}$ to $1.4 M_{\odot}$ (see Table 3 ), whereas for the black histogram to which we compare our predictions, Knödlseder (1997) obtained a total galactic mass of ${ }^{26} \mathrm{Al}$ of $1.8 \pm 0.1 M_{\odot}$. The values listed in Table 3 show that WR stars can account for $20 \%$ to $93 \%$ of the observational estimates of the actual total galactic ${ }^{26} \mathrm{Al}$ mass $\left(1.5-3 M_{\odot}\right)$. The predicted masses are little affected $(\approx 10 \%$ variation) by the uncertainties on the galactic SFR, the IMF slope or the quantity against which the 


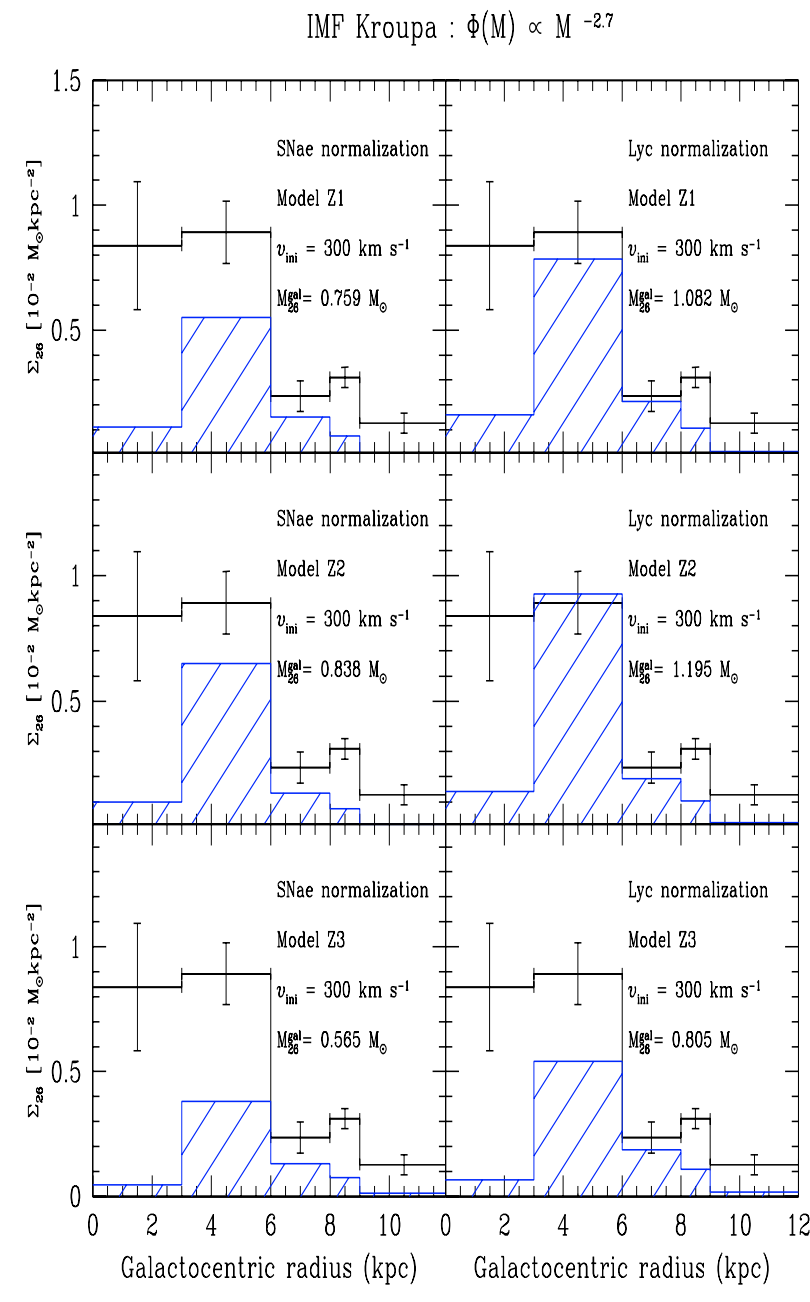

Fig. 8. Same as right column of Figs. 6 and 7 for a Kroupa IMF.

number of massive stars is to be normalized. On the other hand, the predicted total mass of ${ }^{26} \mathrm{Al}$ originating from WR stars varies by as much as $30 \%$ according to the galactic metallicity gradient adopted. The shape of the predicted surface density distributions of ${ }^{26} \mathrm{Al}, \Sigma_{26}$, against the galactocentric radius strongly depends on the SFR adopted, so that the comparison with the values deduced from observations is of little interest.

\section{Conclusion}

We have studied the impact of rotation and initial metallicity on the yields of ${ }^{26} \mathrm{Al}$ ejected by the WR stellar winds. When making use of rotation velocities corresponding to the observed average rotational velocities during the MS phase, i.e. for values around $200 \mathrm{~km} \mathrm{~s}^{-1}$, the inclusion of rotation, all other things being equal, roughly doubles the WR contribution. Higher initial rotation velocities would still increase the quantity of ${ }^{26} \mathrm{Al}$ ejected by the WR stellar winds.

We compared the effects of rotation and those induced by an enhancement of the mass loss rates. On one hand, nonrotating models with enhanced mass loss rates (Meynet et al. 1997) as well as the rotating models presented here (for which the mass loss rates are quite small), are both able to reproduce the observed variation with the metallicity of the number of
WR to O-type stars. On the other hand, the ${ }^{26} \mathrm{Al}$ yields predicted are different, especially in the lower mass domain. This is evidence of the fact that rotation has other more subtle effects rather than an enhancement of the mass loss rates, as already pointed out in Sect. 3. Our rotating models thus predict wind ejected masses that are about a factor of 1.4 superior to the ones provided by Meynet et al. (1997).

We showed that the use of metallicity dependent mass loss rates during the WR phase as suggested by Crowther et al. (2000) does not have a significant impact on the results.

The global contribution of WR stars remains difficult to assess in view of the numerous uncertainties pertaining not only to some physical ingredients of the models as the mass loss rates or the nuclear reaction rates, but also to galactic parameters such as the galactic metallicity gradient or the supernova rate. We showed that the choice of the SFR tracer determines the shape of the ${ }^{26} \mathrm{Al}$ surface density distribution with galactocentric distance, but marginally affects the predicted contributed mass of ${ }^{26} \mathrm{Al}$ by WR. On the other hand, the choice of the metallicity gradient as well as that of the IMF slope may significantly affect the contribution of WR stars to the total galactic mass of ${ }^{26} \mathrm{Al}$, which can amount to $0.6 M_{\odot}$ (shallow metallicity gradient with Kroupa's IMF) up to $1.4 M_{\odot}$ (steep metallicity gradient with Salpeter's IMF).

In that respect the study of associations such as the Cygnus region, sufficiently young to prevent supernovae having much contributed to the ${ }^{26} \mathrm{Al}$ enrichment are of prime importance. Possibly the detection of the $1.809 \mathrm{MeV}$ emission around a single source would be even more important. In that respect the upper limit found for $\gamma^{2}-\mathrm{Vel}$ (Oberlack et al. 2000) already provides a strong constraint. The binary nature of the object however requires some care when comparisons are made with a single star model. This particular case will be studied in detail in a forthcoming paper.

Acknowledgements. A. Palacios acknowledges financial support from ESA PRODEX fellowship No. 90069.

\section{References}

Abbott, D. C., \& Conti, P. S. 1987, ARA\&A, 25, 113

Andrievsky, S. M., Luck, R. E., Martin, P., \& Lépine, J. R. D. 2004, A\&A, 413, 159

Angulo, C., Arnould, M., Rayet, M., et al. 1999, Nucl. Phys. A, 656, 3

Arnould, M., Goriely, S., \& Jorissen, A. 1999, A\&A, 347, 572

Arnould, M., Meynet, G., \& Paulus, G. 1997, A\&A, 321, 452

Bennett, C. L., Fixsen, D. J., Hinshaw, G., et al. 1994, ApJ, 434, 587

Cerviño, M., Knödlseder, J., Schaerer, D., von Ballmoos, P., \& Meynet, G. 2000, A\&A, 363, 970

Clayton, D. D., \& Nittler, L. R. 2004, in Origin and Evolution of the Elements, ed. A. McWilliam, M. Rauch, Carnegie Observatories Astrophysics Series (Cambridge Univ. Press), 4, 300

Crowther, P. S., Smith, L. J., \& Willis, A. J. 1995, A\&A, 304, 269

Crowther, P. A., Dessart, L., Hillier, D. J., Abbott, J. B., \& Fullerton, A. W. 2002, A\&A, 392, 653

Daflon, S., \& Cunha, K. 2003, Proc. IAU Symp., 212, 162

Dearborn, D. S. P., \& Blake, J. B. 1985, ApJ, 288, L21

Denis, R., \& Zahn, J.-P. 1999, A\&A, 347, 734

Diehl, R., Dupraz, C., Bennett, K., et al. 1995, A\&A, 298, 445 
Fliegner, J., \& Langer, N. 1995, in IAU Symp., 163, ed. K. A. van der Hucht, \& P. M. Williams (Dordrecht: Kluwer), 326

Heger, A., \& Langer, N. 2000, ApJ, 544, 1016

Iglesias, C. A., \& Rogers, F. J. 1996, ApJ, 464, 943

de Jager, C., Nieuwenhuijzen, H., \& van der Hucht, K. A. 1988, A\&AS, 72, 259

Knödlseder, J. 1997, Ph.D. Thesis, Paul Sabatier University, Toulouse, France

Knödlseder, J. 1999, ApJ, 510, 915

Knödlseder, J., Cerviño, M., Le Duigou, J. M., et al. 2002, A\&A, 390, 945

Kroupa, P., Tout, C. A., \& Gilmore, G. 1993, MNRAS, 262, 545

Kudritzki, R.-P., \& Puls, P. 2000, ARA\&A, 38, 613

Limongi, M., \& Chieffi, A. 2003, ApJ, 592, 404

MacPherson, G. J., Davies, A. M., \& Zinner, E. K. 1995, Meteoritics, 30,365

Maeder, A. 2002, A\&A, 392, 575

Maeder, A., Grebel, E. K., \& Mermilliod, J.-C. 1999, A\&A, 346, 459

Maeder, A., \& Meynet, G. 2000a, A\&A, 361, 159 (Paper VI)

Maeder, A., \& Meynet, G. 2000b, ARA\&A, 38, 143

Maeder, A., \& Meynet, G. 2001, A\&A, 373, 555 (Paper VII)

Mahoney, W., Ling, J., Jacobson, A., \& Lingenfelter, R. 1982, ApJ, 262,742

Massey, P., \& Johnson, O. 1998, ApJ, 505, 793

McKee, C. F., \& Williams, J. P. 1997, ApJ, 476, 144

Meynet, G., \& Maeder, A. 2005, A\&A, 429, 581 (Paper XI)

Meynet, G., \& Maeder, A. 2003, A\&A, 404, 975 (Paper X)

Meynet, G., \& Maeder, A. 2002, A\&A, 390, 561 (Paper VIII)

Meynet, G., \& Maeder, A. 2000, A\&A, 361, 101 (Paper V)

Meynet, G., Arnould, M., Prantzos, N., \& Paulus, G. 1997, A\&A, 320, 460

Najarro, F. 1999, New Astron. Rev., 44, 213

Najarro, F., Figer, D. F., Hillier, D. J., \& Kudritzki, R. P. 2004, preprint [arXiv: astro-ph/0407188]

Nugis, T., \& Lamers, H. J. G. L. M. 2000, A\&A, 360, 227

Oberlack, U., Wessolowski, U., Diehl, R., et al. 2000, A\&A, 353, 715
Palacios, A., Meynet, G., \& Vuissoz, C. 2004, to appear in the proceedings of the Fifth INTEGRAL Workshop held in February 2004 in Munich, Germany [arXiv: astro-ph/0403706]

Paladini, R., Davies, R. D., \& DeZotti, G. 2004, MNRAS, 347, 237

Plüschke, S., Cerviño, M., Diehl, R., et al. 2002, New Astron. Rev., 46,535

Prantzos, N. 2004, A\&A, 420, 1033

Prantzos, N., \& Aubert, O. 1995, A\&A, 302, 69

Prantzos, N., \& Boissier, S. 2003, A\&A, 406, 259

Prantzos, N., \& Diehl, R. 1996, Phys. Rep., 267, 1

Ramírez, S. V., Sellgren, K., Carr, J. S., et al. 2000, ApJ, 537, 205

Rauscher, T., Heger, A., Hofmann, R., \& Woosley, S. 2002, ApJ, 576, 323

Salpeter, E. E. 1955, ApJ, 121, 161

Scalo, J. M. 1986, Fundam. Cosmic Phys., 11, 1

Scoville, N. Z., \& Sanders, D. B. 1987, H2 in the Galaxy, in Interstellar Processes, ed. D. Hollenbach, \& H. Thronson (Dordrecht: Reidel), 21

Shaver, P. A., McGee, R. X., Newton, L. M., Danks, A. C., \& Pottasch, S. R. 1983, MNRAS, 204, 53

Smith, D. M. 2004a, New Astron. Rev., 48, 87

Smith, D. M. 2004b, to appear in the proceedings of the Fifth INTEGRAL Workshop held in february 2004 in Munich, Germany [arXiv: astro-ph/0404594]

Tammann, G. A., Loeffler, W., \& Schroeder, A. 1994, ApJS, 92, 487

Timmes, F., Woosley, S. E., Hartmann, D., et al. 1995, ApJ, 449, 204 van der Hucht, K. A. 2002, Ap\&SS, 281, 199

Vink, J. S., de Koter, A., \& Lamers, H. J. G. L. M. 2000, A\&A, 362, 295

Vink, J. S., de Koter, A., \& Lamers, H. J. G. L. M. 2001, A\&A, 369, 574

Vuissoz, C., Meynet, G., Knödlseder, J., et al. 2004, New Astron. Rev., 48,7

Wang, B., \& Silk, J. 1994, ApJ, 427, 759

Woosley, S. E., \& Weaver, T. 1995, ApJ, 448, 315 